\title{
A Geometric Derivation of the Probability of Finding a Relay in Multi-rate Networks ${ }^{\star}$
}

\author{
Laura Marie Feeney ${ }^{1}$, Daniel Hollos ${ }^{2}$, Martin Kubisch ${ }^{2}$, Seble Mengesha ${ }^{2}$, and \\ Holger Karl ${ }^{2}$ \\ 1 Swedish Institute of Computer Science, Imfeeney@sics.se \\ 2 Telecommunication Networks Group, TU Berlin, \\ \{hollos, kubisch, mengesha, karl\}@tkn.tu-berlin.de
}

\begin{abstract}
Relaying can improve performance of a wireless network, especially when different transmission modes with distance/cost tradeoffs are available. Examples of such modes include data rates or transmission power. This paper geometrically analyzes the probability that a high-cost direct transmission can be replaced by a combination of low-cost relay transmissions. The main result of the analysis is a technology-agnostic characterization of a communication system's amenability to relaying strategies and some recommendations for how to structure such systems.
\end{abstract}

\section{Introduction}

One way to improve the performance of a wireless system is to replace high-cost transmissions to distant terminals with a sequence of lower-cost transmissions, using intermediate terminals as relays. An example of "cost" is the data transmit rate. In an IEEE $802.11 \mathrm{~b}$ system, a single, long-distance $2 \mathrm{Mbit} / \mathrm{s}$ transmission might be divided into two $11 \mathrm{Mbit} / \mathrm{s}$ transmissions, if a relay is available in the right place, resulting in an (ideal) effective data rate of $5.5 \mathrm{Mbit} / \mathrm{s}$.

Obviously, whether or not a relay is available is a probabilistic question that depends on the distribution of terminals in a cell and on the relationship between the cost of a specific transmission mode and the distances over which it can be used. In the example above, an $11 \mathrm{Mbit} / \mathrm{s}$ transmission can only be used over short distances, limiting the choice of relays.

In practice, whether or not relaying results in performance improvement depends on the cost structure for a particular link technology. For example, there is no benefit to replace a $1 \mathrm{Mbit} / \mathrm{s}$ transmission with a pair of $2 \mathrm{Mbit} / \mathrm{s}$ transmissions. In fact, the overhead cost of transmitting headers twice would certainly result in performance degradation. Accounting for such fixed costs and pertransmission overhead is essential, although analysis of specific link technologies is outside the scope of this paper.

The primary contribution of this work is a geometry-based, analytic model for bounding the performance improvement that can be obtained using intracell relaying. In particular, we develop a closed form expression for the expected

* Corresponding author: Holger Karl. This paper has been partially supported by the German Federal Ministry of Education and Research (BMBF) IBMS2 project. 
proportion of terminals in a cell that can take advantage of relaying, given the terminal density and ranges of two transmission modes. Because it is based on geometric construction, the result generalizes to any link technology and transmission cost metrics, including data rate and energy consumption.

The model makes no assumption about protocol mechanisms for relaying, in effect, assuming that relay terminals are selected without protocol overhead. This idealized result provides a useful basis for evaluating the performance results of some specific relaying protocol. We conclude this paper by proposing a rate adaptive relaying protocol for IEEE 802.11 that exploits the probabilistic insights gained from our analytic work.

\section{Geometric Analysis}

We derive the probability that, for a given terminal, there is another terminal located such that using it as a relay reduces the total cost of communication.

\subsection{Relaying and Network Model}

Consider a wireless cell consisting of an access point and $N$ terminals. Each terminal supports a small number, $k$, of transmission modes $M_{1}, \ldots, M_{k}$. Each mode supports a maximum transmit radius $d_{1}, \ldots, d_{k}$, where $d_{i}<d_{j}$ for $i<j$; $d_{k}$ defines the cell radius. Each transmission mode $M_{i}$ also has associated with it some transmission "cost" $C_{i}$. We assume that there is a direct relationship between distance and cost, thus $C_{i}<C_{j}$ for $i<j$. (The higher the cost, the longer distance can be covered.)

In the IEEE 802.11 rate adaptation example above, "cost" is the time required to transmit a packet. Energy cost, based on variable transmit power, is another example. The discrete cost model is suited to any link technology which provides a small number of discrete transmission modes. Assigning costs for a specific link technology is outside the scope of this work. We assume that transmission mode combinations for which relaying reduces the total cost have been identified.

We do not take into account the impact of relaying on end-to-end packet error rate (PER). As long as the maximum transmit radius for a mode is defined in terms of a relatively low PER and there is only a single relay, we believe that this is an acceptable simplification.

In this work, we only consider the case of a single relay and two transmission modes $(k=2)$. It is fairly straightforward to extend the analysis to larger $k$, although it becomes somewhat cumbersome as the number of cases to be considered grows rapidly; we leave this to forthcoming work. For this restricted problem, we can simplify the notation by normalizing the smaller (low-cost) relay radius $d_{1}$ to 1 and defining the larger (high-cost) transmit radius and cell size $D=d_{2} / d_{1}>1$. We assume that for the link technology in question, it is known that relaying is (at least potentially) cost efficient i.e. that $C_{1}+C_{1}<C_{2}$. 


\subsection{Derivation of the Probability of Relay Utilization}

First, we compute the area $A(r)$ of the region in which a terminal must be located in order to act as a relay for a transmitter located at distance $r$ from the access point. Assuming terminals are uniformly distributed in the cell, it is then straightforward to compute the probability that there is at least one terminal in a region of area $A(r)$. Finally, we compute for each terminal in a cell its expected probability of being able to take advantage of a relay terminal.

Area of the Relay Region. We define $R_{t}(r)$, the relay region of a transmitter $t$ located at distance $r$ from the access point, as the region in which a terminal must be located in order to act as a relay for $t$. The relay region is non-empty only for $1<r \leq \min (D, 2)$. For $0<r \leq 1$, the transmitter is already able to communicate directly with the access point using the lower cost transmission mode. For $r>\min (2, D)$, the transmitter is either too far away to reach the access point via a single relay, or it is outside the cell. ${ }^{1}$

In order for a terminal to act as a relay for a transmitter, the distance between the relay and the transmitter must be less than the relay radius, which is 1 ; otherwise, the transmitter cannot communicate with the relay using the lower cost (smaller transmission radius) transmission mode. Similarly, the distance between the relay and the access point must also be less than the relay radius; otherwise the relay cannot communicate with the access point at the lower cost transmission mode. Figure 1 illustrates this requirement.
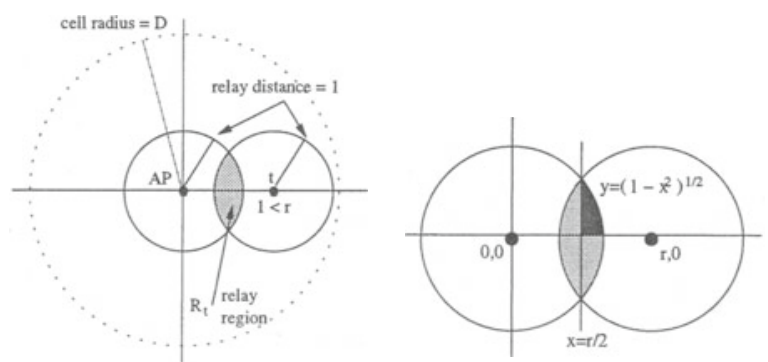

Fig. 1. Relay region $R_{t}(r)$ (left). Detail (right). Without loss of generality, we set the access point at the origin and the transmitter at $(r, 0)$.

We can now compute $A(r)$, the area of the relay region $R_{t}(r)$ for transmitter $t$. Integrating to find the intersection of the unit disks (Figure 1 detail) gives:

$$
A(r)=4 \int_{x=r / 2}^{1} \int_{y=0}^{\sqrt{1-x^{2}}} \mathrm{~d} y \mathrm{~d} x=\pi-r \sqrt{1-\left(\frac{r}{2}\right)^{2}}-2 \arcsin \frac{r}{2} .
$$

${ }^{1}$ The analysis is easily extended to address the use of relays for coverage extension. 
Probability of finding a relay. The probability that a transmitter $t$ is able to take advantage of a relay is equivalent to the probability that there is at least one terminal in its relay region $R_{t}(r)$. (Here we assume an optimal relay protocol.)

Any relay region is, by definition, contained within the cell radius $D$. Assuming terminals are uniformly distributed within the cell, the probability $p$ that a terminal in the cell is in a relay region of area $A(r)$ is therefore $p(r)=\frac{A(r)}{\pi D^{2}}$.

We define $P_{n \geq 1}(r)$ to be the probability that, for some transmitter $t$, at least one of the other $N-1$ terminals is in its relay region $R_{t}(r)$. This is the complement of the probability that all $N-1$ terminals are not in its relay region.

$$
P_{n \geq 1}(r)=1-(1-p)^{N-1}=1-\left(1-\frac{A(r)}{\pi D^{2}}\right)^{N-1}
$$

Expected proportion of terminals finding a relay. To find $P(N, D)$, the expected proportion of terminals in a cell which can take advantage of relaying, we compute the expected value of the probability $P_{n \geq 1}(r)$ for each terminal, which is its expected value over all positions in the cell. The equivalent polar formulation integrates over $r$ the probability that a terminal is located at distance $r$ from the access point and there is at least one terminal in its relay region.

$$
\begin{gathered}
P(N, D)=\lim _{\Delta r \rightarrow 0} \sum_{i=0}^{\left\lfloor\frac{\min (D, 2)}{\Delta r}\right\rfloor}\left(P(\text { terminal in ring from } i \Delta r, \Delta r \text { wide }) P_{n \geq 1}(i \Delta r)\right) \\
=\int_{r=1}^{\min (D, 2)} \frac{2 r}{D^{2}} P_{n \geq 1}(r) \mathrm{d} r=\int_{r=1}^{\min (D, 2)} \frac{2 r}{D^{2}}\left(1-\left(1-\frac{1}{\pi D^{2}} A(r)\right)^{N-1}\right) \mathrm{d} r
\end{gathered}
$$

\subsection{Numeric Solution of $P(N, D)$}

Figure 2 shows the behavior ${ }^{2}$ of $P(N, D)$ for various values of $N$ and $D$. The proportion of terminals that can take advantage of a relay grows rapidly as the Terminal density increases - precisely the situation in which relaying is most needed. There is also a narrow range of optimal values for $D$, which is the ratio between the range of the high and low cost transmission modes.

For the case of rate adaptation in IEEE 802.11 networks, realistic values of $N$ and $D(D \approx 1.25-2.25, N \approx 8-32)$ suggest that between 15 and 55 percent of terminals in a cell can use a relay to increase their effective transmission rate.

\section{Protocol Outline}

We now outline a relaying strategy for rate adaptation in an IEEE 802.11 cell. The proposed technique retains the basic operation of the IEEE 802.11 DCF

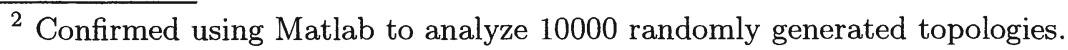




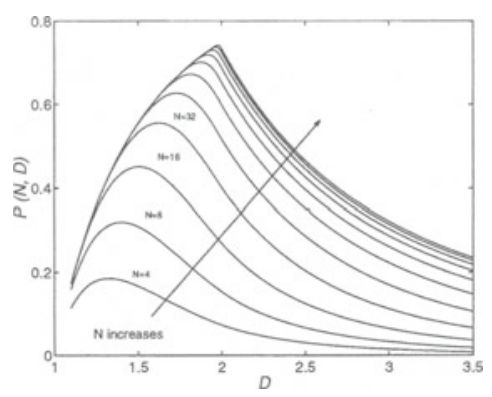

Fig. 2. Behavior of $P(N, D)$ for various $D$ as $N$ becomes large (graph shows curves for $N=4,8,16, \ldots, 4196)$

protocol: RTS and CTS messages are transmitted at the base (lowest) transmission bit rate of the BSS and reserve the channel for the end-to-end duration of the transmission. A terminal that acts as a relay is thus permitted to transmit during this interval without first performing media reservation.

We build upon opportunistic rate adaptation. A terminal transmitting at a low bit rate occasionally attempts a higher bit rate. The terminal will use the RTS/CTS mechanism to reserve the channel for a duration that assumes a sequence of two transmissions at a higher bit rate, plus some backoff interval. The originating terminal then transmits the DATA frame at the higher bit rate.

Any terminal that successfully receives the high-bit-rate frame is a potential relay. It can determine the minimum date rate at which the frame must be retransmitted by comparing the frame duration with the date rate at which the frame was transmitted. For the relayed transmission to succeed only one of the potential relays should actually retransmit the frame and that relay terminal must be able to communicate with the access point at the required data rate.

Each potential relay performs a short random backoff procedure, after which it checks for an already ongoing relay transmission. Thus, there will be a collision only if two relays select the same slot in which to begin their transmissions. The backoff interval can be tuned to reflect the probability of finding a relay, as derived above. The backoff procedure can also be biased such that a relay terminal that is more likely to succeed in communicating with the access point at the higher data rate (e.g. because it has recently done so) selects a shorter backoff interval. The maximum length of the backoff interval must be included in the duration specified in the RTS, allowing a tradeoff between collisions and overhead.

If any of the higher bit rate transmissions fail or if there is no relay available, the originating terminal will not receive an ACK. The terminal simply retransmits at the lower bit rate and continues to transmit at the lower bit rate until it makes another opportunistic attempt to communicate at a higher bit rate.

This approach has the advantage of simplicity and minimal modification to the existing IEEE 802.11 framework. The technique applies directly only to 
traffic on the up-link, although bi-directional down-link traffic can be supported by caching up-link relay information at the access point. The overhead of the backoff interval and risk of collision among relays are potential disadvantages.

\section{Related Work}

The capacity of multi-hop networks has been widely studied. More closely related to the present work are a number of probabilistic studies of the distributions of link distance and path lifetime in multi-hop networks. In particular, Miller derives the probability of a relay connection in a random network as a function of node dispersion [1].

Relaying is frequently proposed to minimize energy consumption in wireless networks. In [2], a geometric model is used to determine for each node which of its neighbors are most efficiently reached directly and which are most efficiently reached via a relay. A distributed algorithm is also presented.

The combination of relaying with rate adaptation has been a motivating example throughout this paper. While this problem has not been extensively studied, a scheme proposed in [3] indicates a significant capacity increase. The impact of multiple transmission rates in an IEEE 802.11 cell is examined in [4].

\section{Conclusion and Future Work}

We have developed a geometric analysis of the probability of finding a suitable relay, using a generic formulation for cost/distance-adaptive transmission modes. For the case of two transmission modes, we determined the expected proportion of terminals in a cell that can take advantage of a relay, as a function of the ratio between the two transmission ranges and the transmitter density in the cell.

We believe that this analysis provides a good general tool for capacity investigations. Further results have already provided insights into the relative suitability for various, multi-rate capable physical layers (IEEE 802.11b and HiperLAN/2). We are also looking at the impact of several transmission modes and a further specification of the relaying protocol outlined here. We expect that the probabilistic understanding of relaying developed here will assist in the design and performance analysis aspects of that work.

\section{References}

1. Miller, L.E.: Probability of a two-hop connection in a random mobile network. In: Proc. 35th Conf. on Information Sciences and Systems (CISS 2001). (2001)

2. Rodoplu, V., Meng, T.H.: Minimum energy mobile wireless networks. IEEE J. on Selected Areas on Communication 17 (1999) 1333-1344

3. Mengesha, S., Karl, H.: Relay routing and scheduling for capacity improvement in cellular WLANs. In: Proc. of Modeling and Optimization in Mobile, Ad Hoc and Wireless Networks (WiOpt'03), Sophia-Antipolis, France (2003)

4. Heusse, M., Rousseau, F., Berger-Sabbatel, G., Duda, A.: Performance anomaly of 802.11b. In: Proc. INFOCOM, San Francisco, CA (2003) 